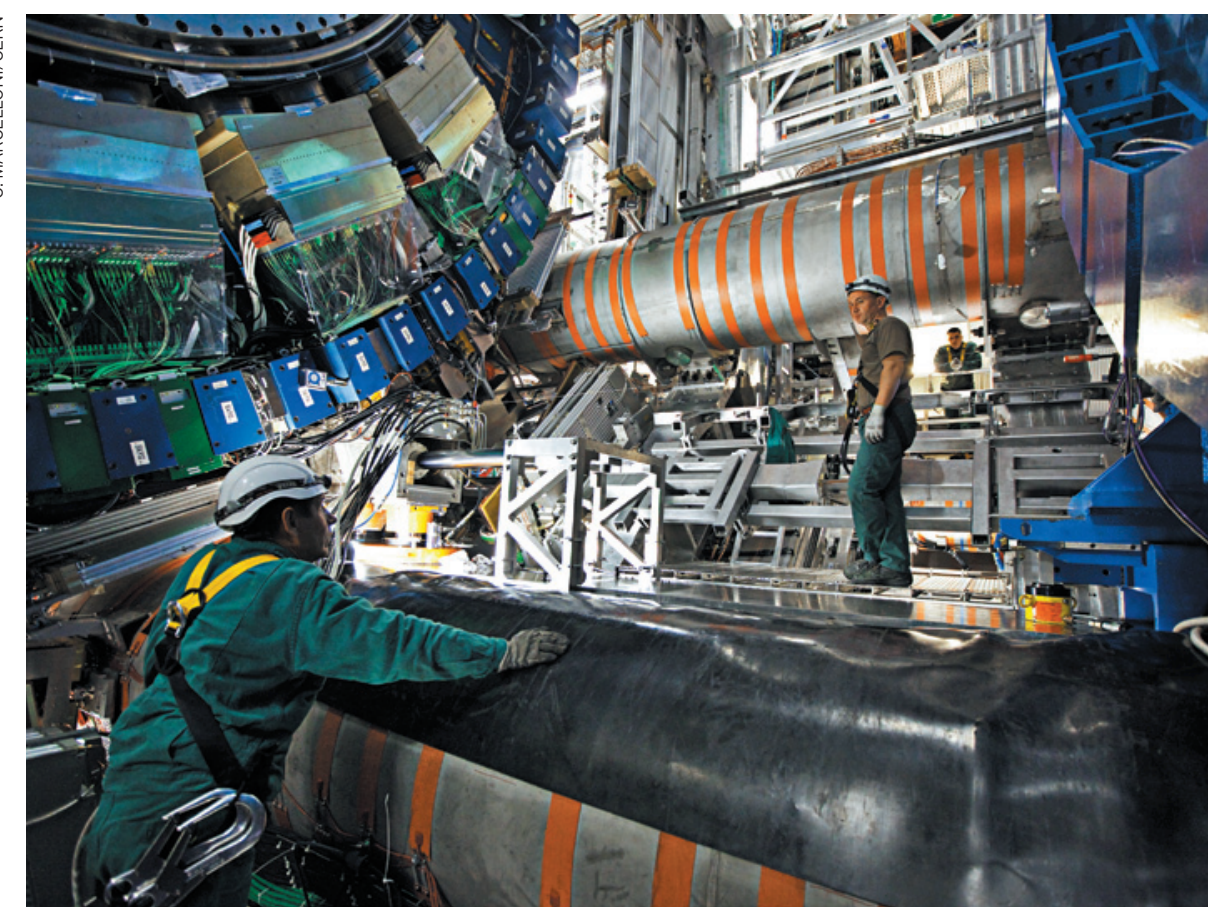

ATLAS at the Large Hadron Collider is one of two experiments focused on finding the Higgs particle.

PARTICLE PHYSICS

\title{
Detectors home in on Higgs boson
}

\section{Hunt gathers momentum as range narrows and hints of apossible signal emerge.}

\section{BY EUGENIE SAMUEL REICH}

$\mathrm{T}$ he result is not definitive evidence of the long-sought Higgs boson - yet. But it is the closest so far to come out of the US\$6.5-billion Large Hadron Collider (LHC) at CERN, Europe's high-energy physics laboratory near Geneva in Switzerland. On 13 December, physicists on the LHC's two largest experiments announced signals consistent with the possible appearance of the Higgs boson, a manifestation of the force field that endows all other particles with mass. If supported by further data, the results suggest a Higgs particle with a mass of about 125 gigaelectronvolts $(\mathrm{GeV})$. But members of both experiments emphasize that the latest data are also statistically consistent with the particle's absence.

"We have not collected enough evidence for a discovery. There is an excess of events compatible with the hypothesis that it could be a Higgs," says Guido Tonelli, spokesman for the Compact Muon Solenoid (CMS) experiment.

His sentiment was echoed by Fabiola Gianotti, spokeswoman for the LHC's ATLAS experiment. "It could well be something intriguing, but it could be a background fluctuation," she says.

Last month, the CMS and ATLAS experiments presented a combined analysis that left little room for the Higgs to hide. If the particle exists, it must have a mass of between $114 \mathrm{GeV}$ and $141 \mathrm{GeV}$. The latest results narrow the field even more: ATLAS has excluded all masses outside the range of $115-130 \mathrm{GeV}$, and the CMS team has revised the range to $117-127 \mathrm{GeV}$. Raising anticipation still further, each experiment separately reports that the LHC's high-energy collisions between protons generated an excess of particles that could be the products of Higgs particle production. The

\section{DNATURE.COM}

For more on the

Large Hadron

Colider see:

www.nature.com/lhc
ATLAS result is consistent with a $125-126 \mathrm{GeV}$ Higgs at a statistical level of at most 3.6 standard deviations, and the CMS team reports a $124-\mathrm{GeV}$ signal of at most 2.6 standard deviations. In particle physics, a statistical significance of five standard deviations is considered to be proof of a particle's existence, and three standard deviations to be evidence that a particle may exist. The ATLAS and CMS results have not yet been combined, so a joint probability is not available.

The situation is further complicated by another signal seen by both experiments at around $119 \mathrm{GeV}$. It is a weaker signal and less convincing as a sign of the Higgs, particularly in the ATLAS data, but its presence has heightened researchers' caution in interpreting what is real and what is not in the new data. A sighting of the Higgs boson at either energy is consistent with the standard model of particle physics, and also with its extension, known as supersymmetry, in which known particles are associated with superheavy partners that have yet to be found.

The Higgs particle is a ripple in the Higgs field, which was proposed in 1964 by several scientists, including British physicist Peter Higgs, to explain why the $\mathrm{W}$ and $\mathrm{Z}$ bosons, which transmit the weak nuclear force, have mass, whereas the photon, which carries the electromagnetic force, does not. Tom Kibble of Imperial College London, another of the theorists to have proposed the Higgs mechanism, says that, over the years, the focus has gradually moved from the Higgs field to spotting the Higgs particle, because all other aspects of the standard model have been observed in particle accelerators. "The only thing missing is the Higgs boson,” Kibble says.

Even if confirmed as an initial sighting of the Higgs particle, the $125-\mathrm{GeV}$ result will leave many questions unanswered. The processes of production and decay of the Higgs particle still need to be carefully defined to help to inform theories beyond the standard model. Such theories would ideally answer long-standing questions such as why the known particles have the masses they do and why gravity is so much weaker than other forces of nature. The latter puzzle is one that supersymmetry is intended to solve.

\section{"There is an excess of events compatible with the hypothesis that it could be aHiggs."}

More data will be needed to establish the existence of the Higgs with confidence. Thus far, the ATLAS and CMS experiments have each detected the results of some 500 trillion proton-proton collisions. Spokespeople say that both experiments should have gathered four times as much data by the end of 2012. "Next year's data should answer it without question," says Bill Murray of the Rutherford Appleton Laboratory near Oxford, UK, who is leading the ATLAS search for the Higgs. Soon enough it will be clear whether this week's hints amount to a seductive mirage or a true glimmer of the LHC's quarry. . 